\title{
The expression and prognostic value of ROCK I and ROCK II and their role in human breast cancer
}

\author{
JANE LANE, TRACEY A. MARTIN, GARETH WATKINS, ROBERT E. MANSEL and WEN G. JIANG \\ Metastasis and Angiogenesis Research Group, University Department of Surgery, \\ Wales College of Medicine, Cardiff University, Heath Park, Cardiff CF14 4XN, UK
}

Received March 11, 2008; Accepted May 2, 2008

DOI: 10.3892/ijo_00000044

\begin{abstract}
The role and expression of ROCKI and ROCKII in human breast cancer was investigated and the effect on clinical outcome assessed. ROCK knockdown cells (MDA-MB$231^{\triangle R O C K I}$ and MDA-MB-231 $\triangle$ ROCKII were tested for their in vitro invasiveness, motility and in vivo tumour growth. Samples of fresh frozen breast tumour tissue $(n=113)$ and normal background tissue $(n=30)$ were processed for immunohistochemical and quantitative RT-PCR analyses. MDAMB-231 ${ }^{\triangle R O C K I}$ and MDA-MB-231 ${ }^{\triangle R O C K I I}$ cells showed significantly decreased invasiveness compared with control cells (mean \pm SEM 4.33 \pm 0.84 MDA-MB-231 ${ }^{\triangle R O C K I} \mathrm{Vs}$. $18.4 \pm 1.4$ control, $\mathrm{p}<0.001 ; 6.8 \pm 1.2$ MDA-MB-231 ${ }^{\triangle R O C K I I}$ vs. $18.4 \pm 1.4$ control, $\mathrm{p}<0.001)$. Similarly, both exhibited reduced motility compared with control cells $(\mathrm{p}<0.001)$ and lost their response to HGF. Importantly, no significant difference existed between knockdown and control cells in in vivo tumour growth. ROCKI was significantly higher in human mammary tumours than normal background tissue $(2.9 \pm 1.1$ vs. $0.29 \pm 0.13, p=0.023$ ), although expression of ROCKII was fairly consistent in both ( $2050 \pm 646$ vs. $2303 \pm 2079)$. ROCKI expression was greater in patients who died from breast cancer than in patients who remained disease free (11.6 $6 \pm 7.1$ vs. $1.95 \pm 0.95)$. Higher levels of ROCKI were associated with increased grade $(0.95 \pm 0.73$ grade $-1 ; 2.11 \pm 1.72$ grade- 2 ; and 4.06 \pm 1.99 grade-3). Levels of ROCKI, but not ROCKII, were significantly correlated with overall survival of patients ( $\mathrm{p}=0.004$, Univariate analysis, median follow-up 120 month).
\end{abstract}

Correspondence to: Dr Jane Lane, Metastasis and Angiogenesis Research Group, University Department of Surgery, Wales College of Medicine, Cardiff University, Heath Park, Cardiff CF14 4XN, UK

E-mail: lanej1@cf.ac.uk

Abbreviations: ROCK, Rho associated serine threonine protein kinase; RT-PCR, real-time polymerase chain reaction; GTPases, guanosine triphosphatases; NPI, Nottingham prognostic index; BSS, balanced salt solution; TBS, tris buffered saline; BSA, bovine serum albumin; HGF, hepatocyte growth factor

Key words: ROCK, Rho, breast cancer, invasion, motility
These results show that ROCKI and possibly ROCKII are key factors in regulation of motility/invasion of breast cancer cells. This, together with significant correlation between ROCKI and poor outcome in clinical breast cancer, indicates that it is a potential target in human breast cancer.

\section{Introduction}

The development of metastasis in breast cancer is a multistep process in which tumour cells undergo changes in their cytoskeletal structure and gene expression leading to changes in cell adhesion, motility and morphology and eventually to metastasis and tissue invasion. A number of these cellular processes are regulated by the Rho GTPases, which function as guanine nucleotide regulated binary switches, affecting cell motility, migration and adhesion primarily by reorganisation of the actin cytoskeletal system (1-3) and by changes in gene expression/transcription (4). Rho proteins have also been shown to play a role in the metastatic phenotype in vivo (5).

It is known that some members of the Rho family proteins are associated with prognosis in bladder cancer (6) and it is thus possible that changes in the activities of the Rho GTPases within the cell could lead to invasion and metastasis. Increased expression of Rho proteins has been demonstrated in tumours from colon, breast and lung, supporting this view (7), together with raised levels of Rho-C, Rho-G and Rho- 6 detected in breast tumour tissue (8).

A number of potential effector proteins have been identified that interact with members of the Rho family which are ultimately responsible for the diverse biological effects of Rho GTPases (9). The Rho-associated serine-threonine protein kinases (ROCKs) are such downstream effectors and the two isoforms ROCK I and ROCK II have been linked to the pathogenesis and progression of several human tumours $(6,10)$. ROCK becomes activated when it selectively binds to the active GTP-bound form of Rho. The action of this Rho/ ROCK signalling pathway has been shown to be associated with tumour progression by regulation of actin cytoskeletal reorganisation (11) and the formation of focal adhesion (12). ROCKs also have an important role in cell migration by enhancing cell contractility and are required for tail retraction of cancer cells (13). In addition, recent work on 3-dimensional models of motility and invasion has identified an important role for Rho/ROCK activity in the amoeboid form of tumour 
Table I. Prognostic indicators and expression of ROCK I and ROCK II assessed by Q-PCR.

\begin{tabular}{|c|c|c|c|c|c|c|c|}
\hline & \multicolumn{2}{|c|}{ Nodal involvement } & \multicolumn{3}{|c|}{ Tumour grade } & \multicolumn{2}{|c|}{ Nottingham prognostic index } \\
\hline & $\operatorname{tnm} 1$ & $\operatorname{tnm} 4$ & Grade 1 & Grade 2 & Grade 3 & NPI1 & NPI3 \\
\hline ROCK I & $2.7 \pm 1.5$ & $30.2 \pm 17$ & $0.95 \pm 0.73$ & $2.11 \pm 1.72$ & $4.06 \pm 1.99$ & $3.6 \pm 1.7$ & $6.6 \pm 5.8$ \\
\hline ROCK II & $3303 \pm 1148$ & $453 \pm 383$ & $4084 \pm 2149$ & $2016 \pm 1148$ & $1474 \pm 753$ & $3090 \pm 1191$ & $381 \pm 124$ \\
\hline
\end{tabular}

Copy number/50 ng RNA.

cell motility. This movement is protease independent and relies upon high activity of Rho, ROCK and MLC2 to generate cortical contractile forces for matrix deformation and is associated with a rounded cellular morphology $(14,15)$.

It has been shown that application of the specific ROCK inhibitors Wf-536 (16) and Y27632 (17,18) produces suppression of tumour growth and metastasis while specific activation of ROCK signalling has been shown to lead to increased tumour cell dissemination (19). In colon cancer, ROCK II has an important role as a critical mediator in cancer cell invasion (20) by modulation of specific matrix metalloproteinases at the site of invadopodia formation. In studies of testicular germ cell tumours (GCT), the protein expression of both ROCK I and ROCK II was found to be significantly higher in tumours than in normal tissue and greater in tumours of higher stage than lower stage $(21,22)$.

The aim of this study was to investigate the role and expression of ROCK I and ROCK II, in human breast tumour tissue and to assess how these levels may affect clinical outcome.

\section{Materials and methods}

Surgical specimens of fresh, frozen breast tissue comprising breast tumours $(n=113)$ and normal background tissue $(n=30)$ were collected during surgery. Information was available on the Nottingham prognostic index (NPI, NPI $<3.4$ indicated patients with predicted good prognosis and is referred to as NPI1 here, NPI =3.4-5.4 with moderate prognosis and referred to as NPI-2, NPI $>5.4$ with poor prognosis and referred to as NPI-3), grade of tumour (grade 1-3), degree tumour staging (TNM 1-4) and clinical outcome for all patients with a mean follow-up period of 72 months (clinical and pathological details are shown in Table I).

Breast cancer cells. Human breast cancer cell lines including MDA-MB-231 were purchased from the European Collection of Animal Cell Cultures (ECACC, Wiltshire, UK) and routinely maintained in DMEM F12 (PAA) supplemented with $10 \%$ foetal calf serum and antibiotics where appropriate.

Ribozyme. Ribozyme transgenes to ROCK I and ROCK II were constructed as we previously reported (23). The following DNA oligos were used to generate ribozymes: for Rock-1: Ctgcaggattcagaacctcaggtctgatgagtccgtgagga and Actagtgtt ggaacaccggattatatattcgtcctcacgga; Ctgcagaggaaaatctaaat caagctgatgagtccgtgagga and Actagtggatggcttaaattccttggtt tcgtcctcacgga. For Rock-2 Ctgcaggtattgcatccagagcaactga tgagtccgtgagga and Actagtttttacactgctgaagttgttttcgtcctca cgga; and Ctgcaggatttcagaacctcaggtctgatgagtccgtgagga and Actagtttggaacaccggattatatttcgtcctcacgga.

Ribozymes were cloned into pcDNA3.1/NT-GFP-TOPO ${ }^{\circledR}$ parent vector (Invitrogen Ltd., Paisley, UK). Vectors were transfected into chemically competent Escherichia coli, and bacterial colonies were grown on agar plates. Colonies were examined using PCR technique to confirm the presence and direction of ribozymes. Plasmid purification and extraction was performed using a plasmid extraction kit (Qiafilter; Qiagen, Crawley, UK). The concentration of each plasmid was determined using a spectrophotometer (WPA UV 1101; Biotech Photometer, Cambridge, UK). MDA-MB-231 human breast cancer cells $(500 \mu 1)$ were mixed with ROCK I ribozyme transgene plasmid, ROCK II ribozyme transgene plasmid or GFP control plasmid and then electroporated at $170 \mathrm{~V}$ with an electroporator (Easyjet; Flowgen, UK). Cells were immediately transferred to complete medium prewarmed to $37^{\circ} \mathrm{C}$ and plated into 6 -well plates. After $24 \mathrm{~h}$ cells were transferred to $25 \mathrm{ml}$ cell culture flasks and selection began with $\mathrm{G} 418$ at $100 \mu \mathrm{g} / \mathrm{ml}$. Medium was changed every 3-4 days. After 4 weeks, cells were changed to maintenance medium (with $25 \mu \mathrm{g} / \mathrm{ml}$ G418). ROCK I knockdown cells were designated MDA-MB-231 ${ }^{\triangle R O C K I}$ and ROCK II knockdown MDA-MB-231 ${ }^{\Delta R O C K I I}$.

Immunohistochemistry. Frozen sections of breast tumour and background tissue were cut at a thickness of $6 \mu \mathrm{m}$ using a cryostat. The sections were mounted on super frost plus microscope slides, air dried and then fixed in a mixture of $50 \%$ acetone and $50 \%$ methanol. The sections were then placed in 'Optimax' wash buffer for 5-10 min to rehydrate and were then incubated for $20 \mathrm{~min}$ in a $0.6 \%$ BSA blocking solution and probed with the either the ROCK I (sc-17794) or ROCK II (sc-5561) primary antibody (Santa Cruz Biotechnology Inc., Santa Cruz, CA, USA). Following extensive washings, sections were incubated for $30 \mathrm{~min}$ in the secondary biotinylated antibody (Multilink Swine antigoat/mouse/rabbit immunoglobulin, Dako Inc.). Following washings, Avidin Biotin Complex (Vector Laboratories) was then applied to the sections followed by extensive washings. Diamino benzidine chromogen (Vector Labs) was added to the sections which were incubated in the dark for $5 \mathrm{~min}$. Sections were then counterstained in Gill's haematoxylin and dehydrated in ascending grades of methanol before clearing in xylene and mounting under a cover slip. 
Immunofluorescence. Two chamber slides were prepared by pipetting $200 \mu \mathrm{l}$ of DMEM F12 medium into each well and leaving for 10-15 min. MB-MDA-231, MDA-MB-231 ${ }^{\triangle R O C K I}$ (knockdown), MDA-MB-231 $\triangle$ ROCKII (knockdown), control, and wild-type cells were then aliquoted into the wells and left in the incubator for $1 \mathrm{~h}$ to form a confluent monolayer. Cells were fixed using ice cold ethanol followed by rehydration in BSS buffer and permeabilised in $0.1 \%$ Triton X-100 for $60 \mathrm{sec}$ followed by a further wash in BSS buffer. One slide was washed in BSS buffer and mounted in FluorSave ${ }^{\mathrm{TM}}$ (Calbiochem) and stored in foil at $4^{\circ} \mathrm{C}$. The second slide was blocked with $10 \%$ horse serum in TBS for 40 min followed by $100 \mu \mathrm{l}$ of a 1:500 dilution of primary antibody to ROCK I or ROCK II in 3\% horse serum for $1 \mathrm{~h}$. Cells were then washed in $3 \%$ horse serum and $100 \mu \mathrm{l}$ of secondary antibody was added to each well and left for $1 \mathrm{~h}$. The secondary antibody was prepared using a 1/500 dilution of anti-rabbitFITC in 3\% horse serum. Cells were then washed in 3 changes of $3 \%$ horse serum, mounted in FluorSave ${ }^{\mathrm{TM}}$ and stored in foil as described above until viewed using an Olympus BX51 Fluorescence microscope with Hamamatsu Orca ER digital camera.

In vitro invasion assay. Trans-well chambers equipped with $6.5 \mathrm{~mm}$ diameter polycarbonate filter (pore size $8 \mu \mathrm{m}$ ) (Becton-Dickinson Labware, Oxford, UK) were pre-coated with $50 \mu \mathrm{g} /$ membrane $(100 \mu \mathrm{l})$ of solubilised basement membrane in the form of Matrigel (Collaborative Research Products, Bedford, MA) and dried overnight.

After membrane re-hydration (100 $\mu$ l complete medium), 30,000 cells were aliquoted into each insert with/without HGF (25 ng/ml). After 96-h co-culture, non-invasive cells were removed from the inner chamber with a cotton swab. Invaded cells on the underside of the insert were then fixed (4\% formaldehyde) and stained with crystal violet. The cells were then counted microscopically (20 fields/insert).

Invasion assay was also carried out with and without ROCK inhibitor Y-27632 (sc-3536, Santa Cruz Biotechnology).

Cytodex-2-bead motility assay. Cells were pre-coated onto cytodex-2 carrier beads (Sigma) for $2 \mathrm{~h}$ in complete medium. After the medium was aspirated and the cells washed (twice in complete medium), they were aliquoted into wells of a 96-well plate in triplicate $(300 \mu \mathrm{l} /$ well). HGF $(25 \mathrm{ng} / \mathrm{ml})$ was added and the cells incubated overnight. The beads were washed off in medium, and the cells that had migrated onto the floor of the well fixed (4\% formaldehyde) and stained with crystal violet. The cells were then counted microscopically.

In vitro growth assay. Cells were counted with a haemocytometer counting chamber and a specific number of cells (4,000 per well) were seeded into each well of a 96-well plate with culture medium (DMEM). The culture plate was then incubated at $37^{\circ} \mathrm{C}$ for up to 5 days followed by aspiration of the medium, fixation of the cells with $4 \%$ formalin $(100 \mu \mathrm{l} /$ well) and thorough rinsing in tap water. Cells were stained with $200 \mu \mathrm{l}$ crystal violet for $20 \mathrm{~min}$ and thoroughly rinsed in tap water. The plate was left to dry for $2-3 \mathrm{~h}$ followed by extraction of the dye with $10 \%$ acetic acid (100-200 $\mu 1$ per well). The plate was then read spectrophotometrically at a wavelength of $540 \mathrm{~nm}$ using a multi-well plate reader (BioTek).

RNA extraction and RT-PCR. RNA was isolated from breast cancer cell lines, MDA-MB-463, MDA-MB-435S, MDA-MB-231, MDA-MB-436, MCF-7, ZR-751, MDA-MB468, BT-482, BT-474, using a standard RNA-zol procedure. Between 20-30 frozen sections of each mammary tissue sample were first homogenised in RNA extraction buffer before extraction. For RT-PCR, cDNA was synthesised as described in the manufacturer's protocol (ABgene Reverse Transcription System, ABgene, Surrey, UK). The cDNA obtained was amplified by a standard PCR mixture (as supplied in Pre-aliquoted Reddy-Load Mix, Advanced Biotechnologies). Cycling conditions for the $25 \mu 1$ reaction mix were $94^{\circ} \mathrm{C}$ for $4 \mathrm{~min}$, followed by 36 cycles of $94^{\circ} \mathrm{C}$ for $15 \mathrm{sec}, 55^{\circ} \mathrm{C}$ annealing for $15 \mathrm{sec}$ and $72^{\circ} \mathrm{C}$ for $30 \mathrm{sec}$, followed by a final extension of $7 \mathrm{~min}$ at $72^{\circ} \mathrm{C}$. Q-PCR for $\beta$-actin was also performed on the same samples to correct for any residual differences in the initial level of RNA in the specimens. The products were visualised on a $0.8 \%$ agarose gel following staining with ethidium bromide.

Quantitative-PCR analysis. The Q-PCR system used the Amplifluor $^{\mathrm{TM}}$ Uniprimer ${ }^{\mathrm{TM}}$ system (Intergen Company Oxford, UK) and Thermo-Start ${ }^{\circledR}$ (ABgene, Epsom), as we previously reported (8). Specific primers were designed by the authors and manufactured by Invitrogen (Invitrogen Life Technologies, Paisley, Scotland, UK). Using the Icycler IQ system (Bio-Rad, Hemel Hempstead, UK), which incorporates a gradient thermocycler and a 96-channel optical unit, the plasmid standards and breast cancer cell cDNA were simultaneously assayed in duplicated reactions with the following conditions: enzyme activation at $95^{\circ} \mathrm{C}$ for $12 \mathrm{~min}$, 1 cycle, followed by 60 cycles of denaturing: $95^{\circ} \mathrm{C}$ for $15 \mathrm{sec}$; annealing: $55^{\circ} \mathrm{C}$ for $40 \mathrm{sec}$; extension: $72^{\circ} \mathrm{C}$ for $25 \mathrm{sec}$. Using purified plasmids as internal standards, the level of each molecule cDNA (copies/50 ng RNA) in the breast cancer samples were calculated. Q-PCR for B-actin was also performed on the same samples, to correct for any residual differences in the initial level of RNA in the specimens (in addition to spectrophotometry). The products of Q-PCR were verified on agarose gels. Primer pairs for Q-PCR were as follows: ROCK I F1 (5'-cccagcagatgatcaagtat); ROCK I ZR (5'-actgaacctgaccgtacatgtcatctcagttgaattc); ROCK II F1 (5'-catatggacaaaaaggagga; ROCK II ZR (5'-actgaacetgaccgt acactgctcttgtgtagaatttgc).

In vivo development of mammary tumours. This was based on a similar method recently described (23). Athymic nude mice (Nude CD-1) of 4-6-weeks old were purchased from B\&K Universal Ltd (Aldbrough, Hull, England), and maintained in filter-toped units. Rock manipulated breast cancer cells, control plasmid transfected cells or wild-type cells in suspension (in $0.5 \mathrm{mg} / \mathrm{ml}$ Matrigel) were injected subcutaneously at the left scapula area. Mice were weighed and tumour sizes measured twice weekly for 4 weeks. Mice with weight loss over $25 \%$ and tumour size larger than $1 \mathrm{~cm}$ 

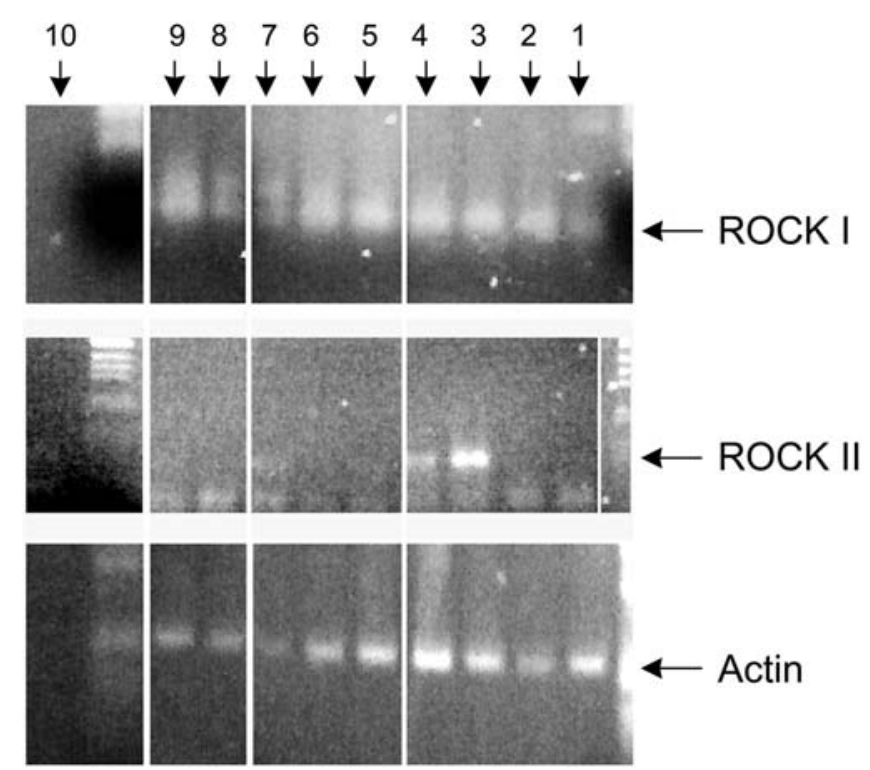
1. MDA-MB-463
6. ZR-751
2. MDA-MB-453S
7. MDA-MB-468
3. MDA-MB-231
8. BT-482
4. MDA-MB-436
5. MCF-7
9. BT-474
10. Negative control

Figure 1. RT-PCR showing expression of ROCK I and ROCK II in human breast cancer cells. $\beta$-actin for standardization.

in any dimension were terminated according to the UK Home Office and UKCCCR guideline. The volume of the tumor was determined using the formula: tumour volume $=0.523 \mathrm{x}$ width $^{2} \mathrm{x}$ length (24). At the conclusion of the experiment, animals were terminally anaesthetised; primary tumours were dissected, weighed and frozen at $-80^{\circ} \mathrm{C}$. Part of the primary tumours was fixed for histological examination.

Statistical analysis. Statistical analysis was performed with MINITAB version 11.2 (Minitab Inc., State College, PA) using two sample Student's t-tests.

Survival curves. In order to assess the long-term survival rates of patients with high and low levels of ROCK I and ROCK II the overall survival data were analysed to produce Kaplan-Meier survival curves.

\section{Results}

Expression of ROCK I and ROCK II in human breast cancer. Initially we investigated the expression of both ROCK I and II in a range of human breast cancer cell lines using RT-PCR (Fig. 1). ROCK I was expressed in all of the nine cell lines studied while ROCK II expression was seen predominantly in the MDA-MB-436 and MDA-MB-231 cell lines. The expression of both ROCK isoforms was seen most strongly in the MDA-MB-231 cells, (ROCK II expression being higher than ROCK I) and these were subsequently selected for use in the functional assay experiments following generation of ROCK knockdown cells (see below).
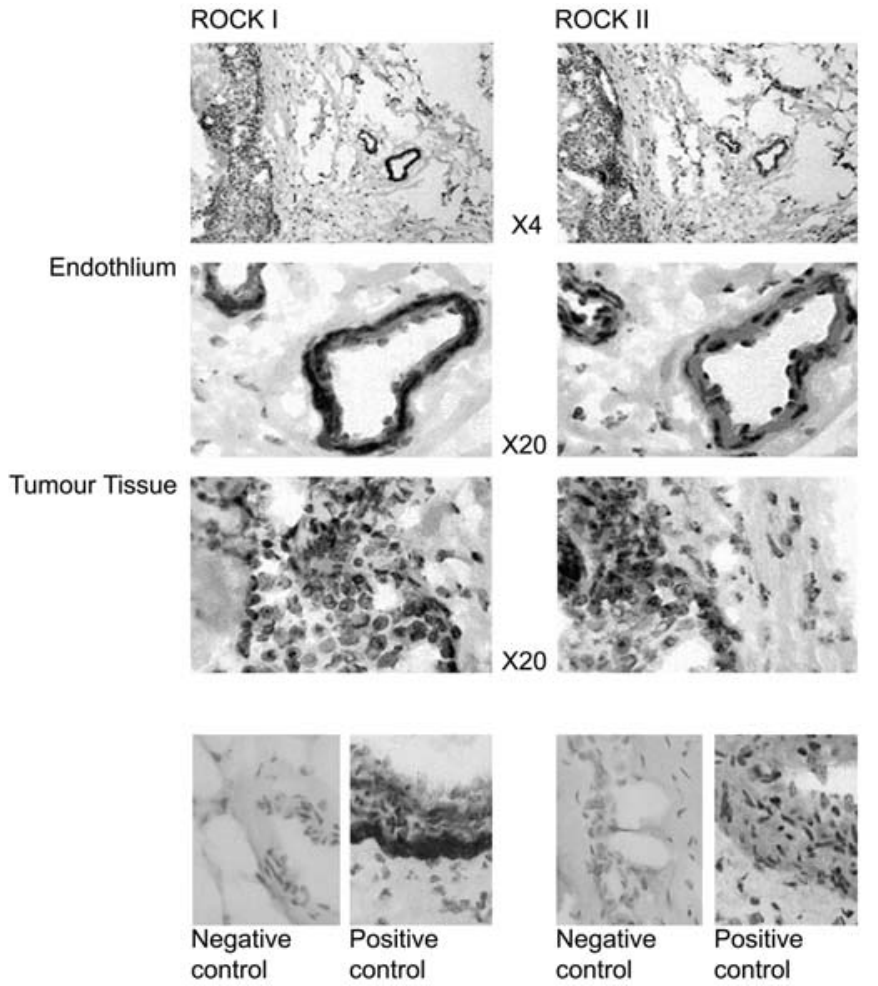

Figure 2. Immunohistochemical staining of breast tumour tissue showing difference in intensity of staining of ROCK I (left panel) and ROCK II (right panel). The top photographs (X4) show a typical section from a patient with breast cancer showing both breast tumour tissue and associated normal endothelium. Also shown at higher magnification (X20) can clearly be observed the differences in staining between endothelium and tumour tissue for both ROCK I and ROCK II. Bottom panel shows positive and negative controls for the ROCK antibodies.

Immunohistochemical staining of tumour tissue showed a more intense staining for ROCK I than for ROCK II (Fig. 2). The level of ROCK I transcripts seen in tumour tissue was significantly higher than that exhibited in normal background tissue $(2.9 \pm 1.1 ; 0.29 \pm 0.13$ respectively, $\mathrm{p}=0.023)$, although the expression of ROCK II was consistent in both tumour and normal breast tissue $(2083 \pm 642 ; 2303 \pm 2079$ respectively, $\mathrm{p}=0.92)$ (Fig. 3A).

ROCK I and II expression and clinical outcome. ROCK I expression was found to be much greater in patients who died from breast cancer than in those patients who were alive and well at the end of the follow-up period (11.6 \pm 7.1 ; $1.95 \pm 0.95$ respectively, $\mathrm{p}=0.20$ ) with the converse true for ROCK II expression $(153 \pm 89$; 2231 \pm 765 , p=0.008) (Fig. 3B). This pattern of increase in ROCK I levels with a corresponding decrease in ROCK II expression was also seen in relation to tumour staging $(2.7 \pm 1.5 \mathrm{TNM} 1$ vs. $30.2 \pm 17$ TNM4 ROCK I, $\mathrm{p}=0.20)$ and $(3303 \pm 1148$ TNM1 vs. $453 \pm 383$ TNM 4 ROCK II, p=0.02), (Fig. 3C), and with an increase in grade of tumour from grade 1 through to grade $3(0.95 \pm 0.73$; $2.11 \pm 1.72 ; 4.06 \pm 1.99$ for ROCK I, p=0.15) and (4084 \pm 2149 ; 2016 \pm 1148 ; 1474 \pm 753 for ROCK II, p=0.52 ) (Fig. 3D). A significant decrease in ROCK II expression in relation to NPI was observed $(3090 \pm 1191$ for NPI 1 vs. $381 \pm 124$ for NPI 3 $\mathrm{p}=0.027)$ together with an increase in ROCK I expression (3.6 \pm 1.7 NPI 1 vs. $6.6 \pm 5.8$ NPI 3, p=0.62) (Fig. 3E). 

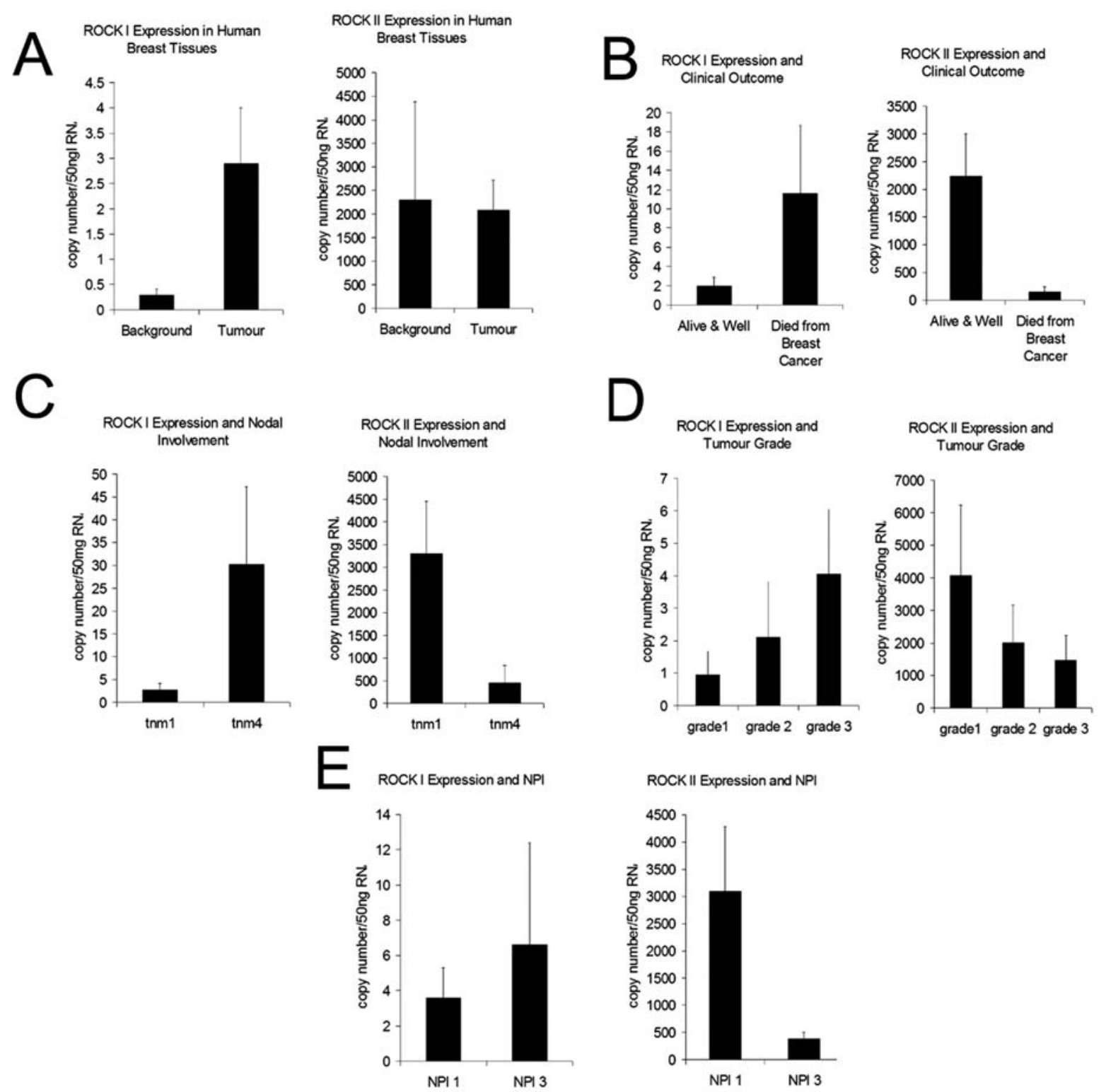

Figure 3. The differences in expression of ROCK I (left) and ROCK II (right) at the RNA level and different clinical parameters. (A) The expression levels of the background and tumour samples; (B) Correlation of ROCK I and ROCK II expression with clinical outcome of the patients; (C) ROCK I and ROCK II expression in relation to nodal involvement; (D) Tumour grade with ROCK I and ROCK II levels of expression; (E) differences in expression of ROCK I and ROCK II with increased NPI.

Manipulation of ROCK I and ROCK II expression and the impact on motility and invasion, in vitro. We went on to generate ROCK knockdown cells by way of ribozyme transgenes. The presence of the transgenes in MDA-MB-231 cells was confirmed by fluorescence: the GFP signal could clearly be observed in the transfected cells (Fig. 4A). The wild-type cells showed little fluorescence in comparison. Immunofluorescence staining of WT MDA-MB-231 cells, MDAMB-231 ${ }^{\triangle R O C K I}$ and MDA-MB-231 ${ }^{\triangle R O C K I I}$ cells also confirmed knockdown of ROCK I and ROCK II respectively (Fig. 4B). Immunofluorescence staining of MDA-MB-231 cells was also employed to look at F-actin and ROCK I distribution during treatment with HGF, Y27632 and a combination of HGF and the inhibitor. Both F-actin and ROCK I were seen to be localised at the cell periphery and in the perinuclear region in the wild-type cells. During treatment with HGF the ROCK I staining was intensified in the perinuclear region with reduced F-actin staining around the nucleus. Use of the ROCK inhibitor (Y27632) eliminated ROCK I expression as did the combination of HGF and Y27632. The response of F-actin to the ROCK inhibitor alone and in combination with HGF was to localise in the perinuclear region of the cells (Fig. 4C). The success of knocking down both ROCK I and ROCK II in the MDA-MB-231 cells was further confirmed by Western blotting (Fig. 5).

MDA-MB-23 $1^{\triangle R O C K I}$ and MDA-MB-231 ${ }^{\triangle R O C K I I}$ cells had significantly decreased invasiveness compared with MDA-MB-231 wild-type cells $(4.33 \pm 0.84$ for MDA-MB$231^{\triangle R O C K I}$ vs. $20.7 \pm 1.2$ for control, $\mathrm{p}<0.01 ; 6.8 \pm 1.2$ for MDAMB-231 ${ }^{\triangle R O C K I I}$ vs. 20.7 \pm 1.2 for control, $\mathrm{p}<0.006$ ) (Fig. 6A). MDA-MB-231 $1^{\triangle R O C K I}$ and MDA-MB-231 $1^{\triangle R O C K I I}$ cells showed reduced response to hepatocyte growth factor/scatter factor, in their invasiveness $(5.20 \pm 0.58$ vs. $4.33 \pm 0.84$, ROCK I $\mathrm{p}>0.09$; with and without HGF, respectively and 5.2 \pm 0.58 
A
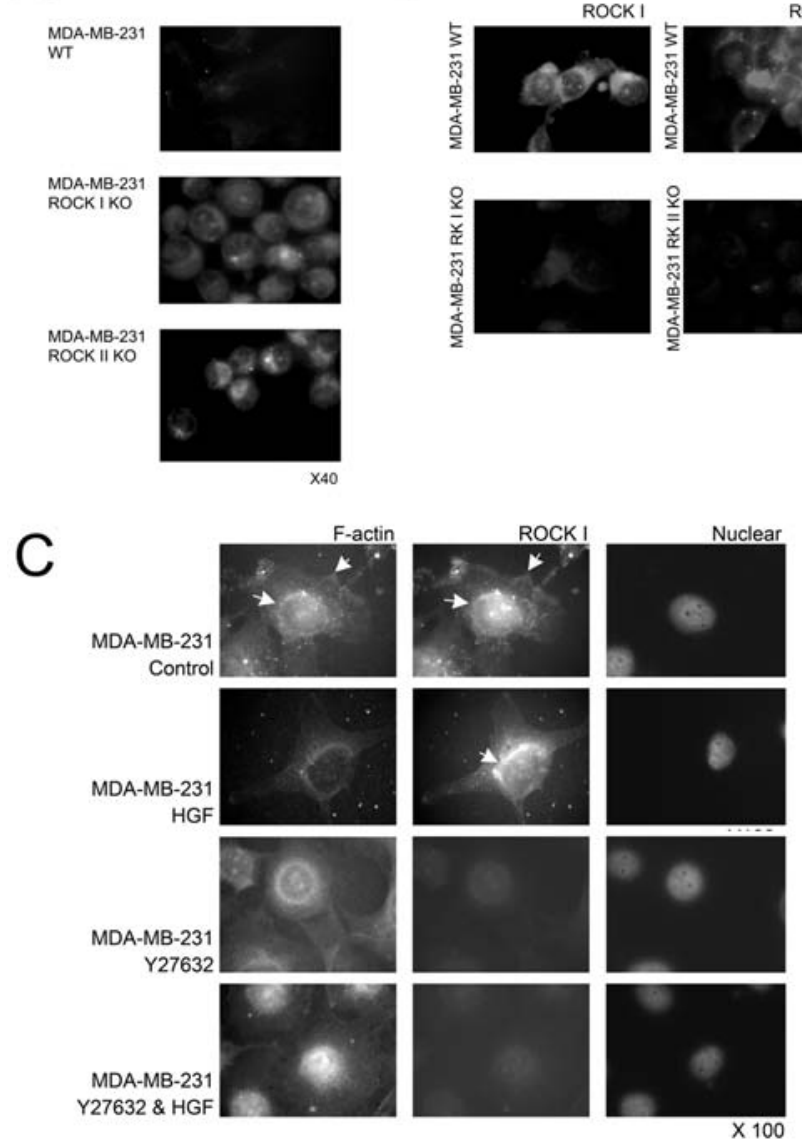

Figure 4. Immunofluoresence staining of (A) MDA-MB-231 WT, MDA-MB$231^{\triangle R O C K I}$ and MDA-MB-231 $\triangle$ ROCKII cells. This figure confirms the presence of the plasmids transfected into MDA-MB-231 cells; (B) MDA-MB-231 WT cells stained with ROCK I or ROCK II antibody (top panel); MDAMB-231 $1^{\triangle R O C K I}$ and MDA-MB-231 $1^{\triangle R O C K I I}$ (bottom panel) confirming knockout of ROCK I and ROCK II. Immunofluorescence staining of MDAMB-231 WT cells to show localization of F-actin and ROCK during treatment with HGF, the ROCK inhibitor Y27632 and a combination of both HGF and Y27632 (C).

vs. $6.8 \pm 1.2$, ROCK II p<0.01) compared with wild-type MDA-MB-231 which responded to HGF with increased invasiveness $(29.0 \pm 1.7$ vs. $23.2 \pm 2.3$, $\mathrm{p}=0.07$; with and without HGF, respectively). Both knockdown cells had reduced their motility compared with control cells $(0.25 \pm 0.25$ for MDA-MB-231 $1^{\triangle R O C K I}, 0.25 \pm 0.25$ for MDA-MB-231 $1^{\triangle R O C K I I} \mathrm{vs.}$ $62 \pm 3.8$ for control, $\mathrm{p}<0.001$ ) (Fig. 6B).

ROCK I and ROCK II knockout did not affect the in vivo growth. The impact of knocking down the expression of either ROCK I or ROCK II on tumour growth was tested using an in vivo model. MDA-MB-231 ${ }^{\triangle R O C K I}$, MDA-MB-231 $\triangle$ ROCKII cells, tranfection control and wild-type MDA-MB-231 cells were used in the athymic nude mouse model. Over the period of 4 weeks, we did not observe a significant difference between these groups. The tumour volume for the wild-type cells was $390.7 \pm 185.6 \mathrm{~mm}^{3}$, compared with $298.5 \pm 186.2 \mathrm{~mm}^{3}$ for MDA-MB-231 ${ }^{\triangle R O C K I}$ tumours, $402.1 \pm 308.7 \mathrm{~mm}^{3}$ for MDAMB-231 ${ }^{\triangle R O C K I I}$ and $431.8 \pm 247.4 \mathrm{~mm}^{3}$ for transfection control ( $>0.05$ between all the groups) (Fig. 6C). The transplant
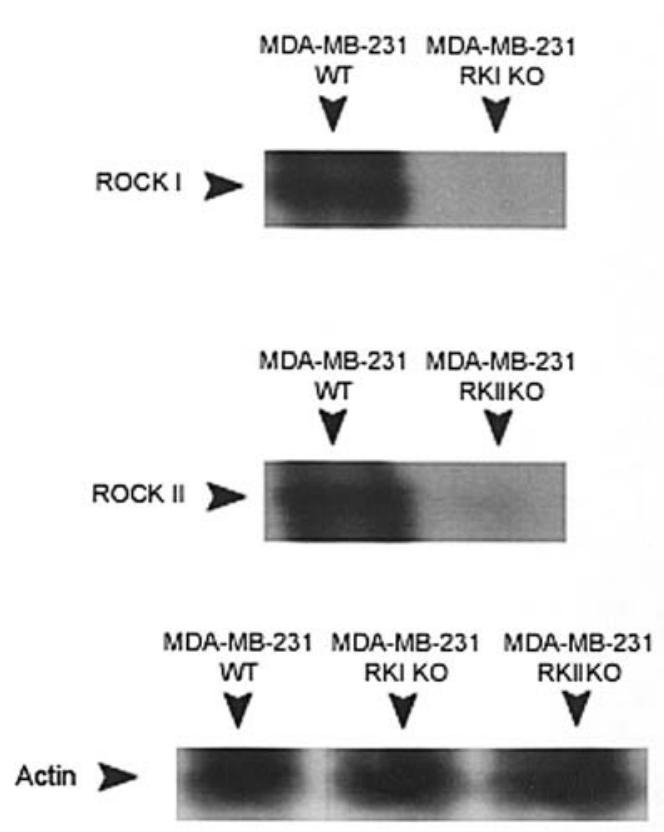

Figure 5. Western blotting to confirm knockdown of ROCK I and ROCK II expression together with loading control.

tumour tissue was dissected and immunohistochemically stained using ROCK I, ROCK II and BCSG-1 antibodies. The staining shows a reduction in the level of both ROCK I and ROCK II in the ribozyme transfected tumour tissue 4 weeks after transplant compared with wild-type tumour tissue. The BCSG breast cancer marker was used to determine that the tumours were indeed human breast cancer cells and not mouse tissue (Fig. 7).

Effect of ROCK inhibitor. We further tested the effects of a ROCK inhibitor (Y27632) on the cells. MDA-MB-231 GFP control cells treated with the inhibitor showed a significant decrease in invasiveness when compared with those untreated cells $(78.6 \pm 6.42$ vs. $131.6 \pm 20.8 \mathrm{p}<0.005)$. Treating the MDA-MB-231ROCK ${ }^{\triangle R O C K I}$ cells with the ROCK inhibitor had a negligible effect on invasion $(43.0 \pm 8.83$ vs. $45.5 \pm 6.43$ with and without inhibitor respectively $\mathrm{p}=0.48)$. Untreated MDA-MB-231-GFP control cells also exhibited greater invasiveness compared with untreated MDA-MB-231 $\triangle$ ROCKI cells (131.6 \pm 20.8 vs. $45.5 \pm 6.43$ p<0.005) (Fig. 8).

ROCK I and ROCK II expression correlated with long-term survival. To determine whether ROCK I and ROCK II transcript levels were associated with long-term survival, we divided patients into those with high levels and those with low levels of ROCK I and II. The cut-off point was determined using the Nottingham prognostic index, and was set at the level at which patients had moderate prognoses (Nottingham Prognostic Index 3.4-5.4). As shown in the Kaplan-Meier survival curve (Fig. 9), high levels of ROCK I correlated significantly with shorter overall survival; mean survival 103.20 months (74.37-132.03 months 95\% CI) vs. 139.87 months (130.31-149.43 months 95\% CI) for those with low levels of ROCK I; $\mathrm{p}=0.0304$. High levels of ROCK II also correlated with shorter overall survival, mean survival 


\section{A Invasion of Human Breast Cancer Cell Line MDAMB- 231 with and without $\mathrm{HGF}(25 \mathrm{ng} / \mathrm{ml})$}

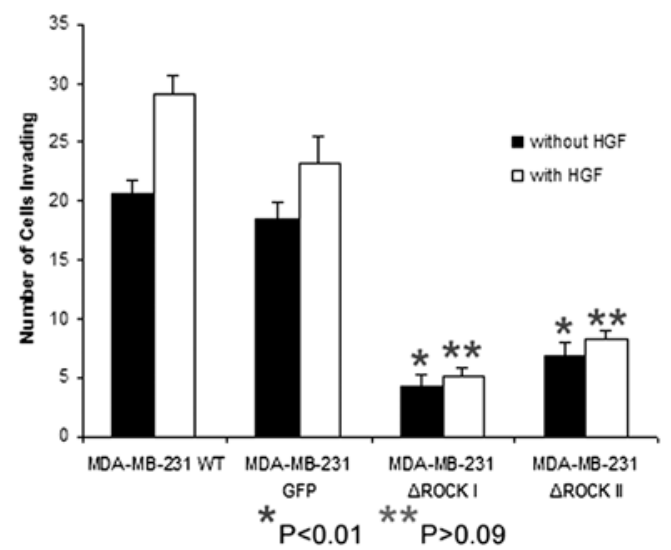

C In Vivo Tumour Growth over 4 weeks

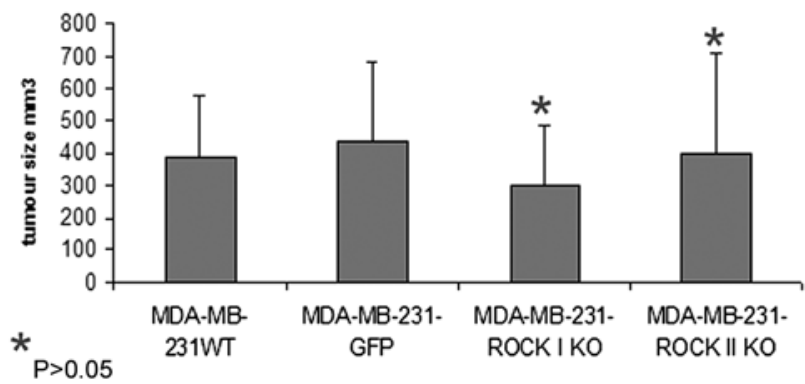

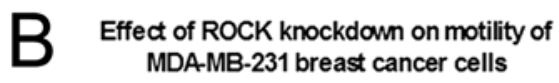

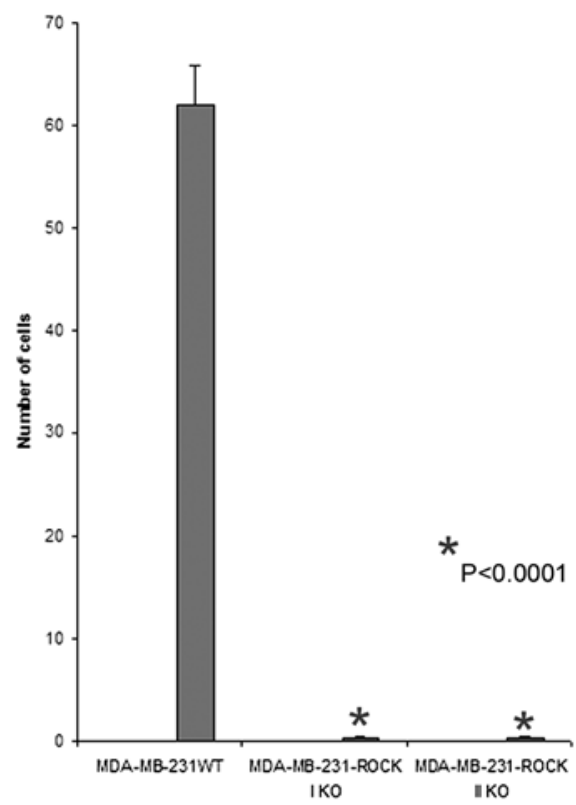

Figure 6. Graphical representation of the difference in invasiveness of MDA-MB-231 ${ }^{\triangle R O C K I}$ and MDA-MB-231 $\triangle$ ROCKII cells compared with wild-type control MDA-MB-231 cells and in response to treatment with HGF (A). Graph to show the effect of ROCK knockdown on the motility of MDA-MB-231 breast tumour cells (B). Graph showing the in vivo tumour growth of ROCK knockdown MDA-MB-231 cells (C).
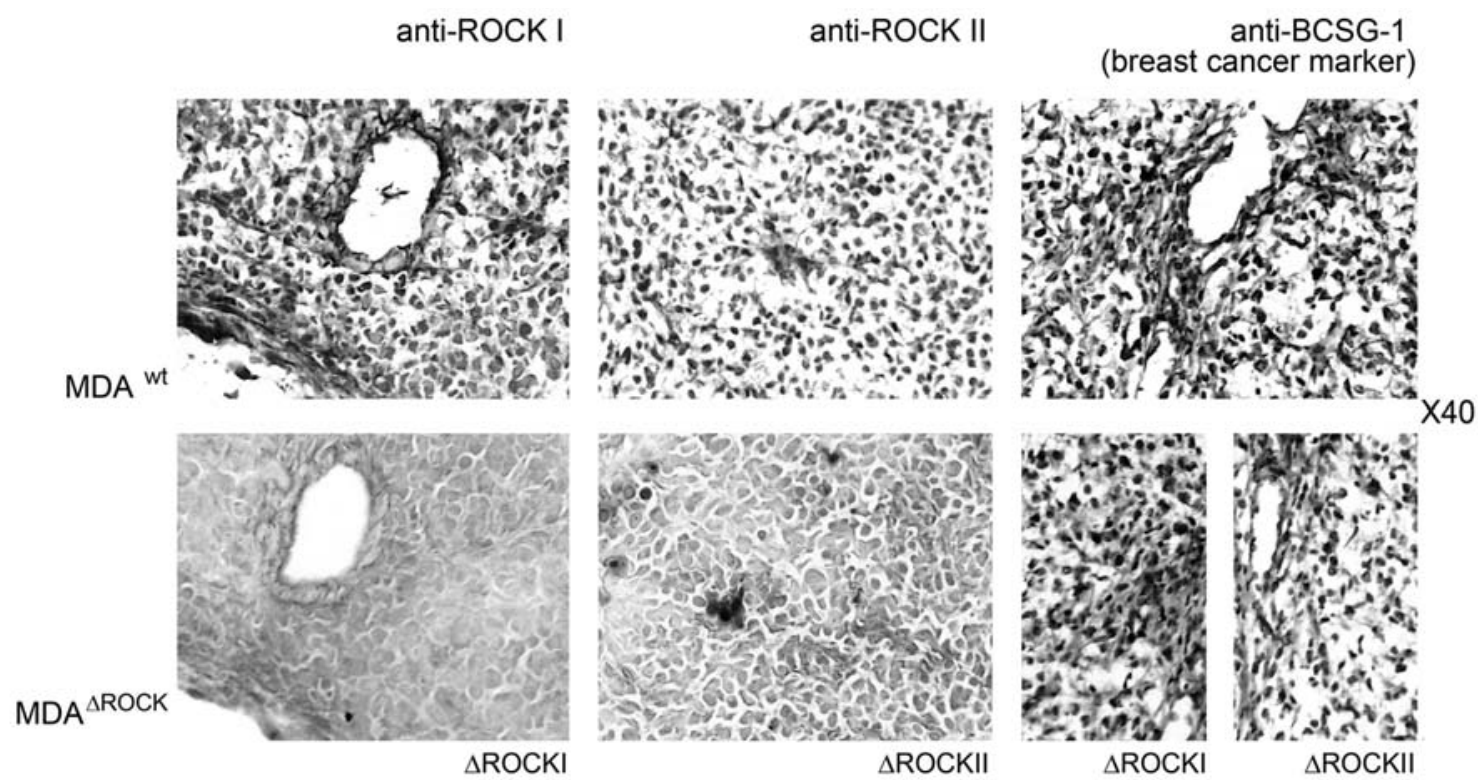

Figure 7. Immunohistochemical staining of transplant tumour tissue 4 weeks after transplant, showing reduction of ROCK I and II expression. BCSG-1 human breast cancer marker used to confirm tissue origin.

125.24 months (105.78-144.7 months $95 \%$ CI) vs. 133.81 months (123.04-144.58 months $95 \%$ CI) for those with low levels of ROCK II, although this did not reach a level of significance $(\mathrm{p}=0.4550)$.

\section{Discussion}

It appears from the analysis of gene transcript that ROCK I and ROCK II behave differently within the breast tumours. 


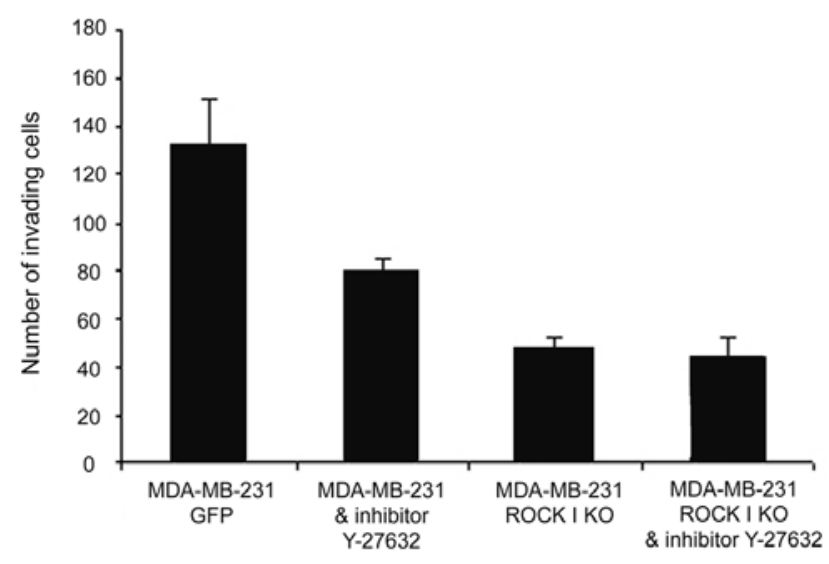

Figure 8. The effect of the ROCK inhibitor Y-27632 on MDA-MB-231 control plasmid cells which reduced their invasiveness when treated with the inhibitor. Treatment of MDA-MB-231 ${ }^{\text {AROCKI }}$ cells with Y-27632 had negligible effect.

Expression of ROCK I, at both mRNA and protein level, is much higher in tumour tissue compared with normal tissue, and is also more highly expressed in later stages of the disease and in patients with poor prognosis in relation to tumour staging, grade and Nottingham prognostic index. Conversely, ROCK II levels do not seem to vary significantly between normal and tumour tissue, although a significant decrease is seen in ROCK II mRNA levels in patients who died from breast cancer and in relation to tumour staging and the Nottingham prognostic index. This would indicate that the two isoforms of ROCK have different roles to play within Rho-mediated cell signalling, with low levels of ROCK II being as important for tumour cells as high levels of ROCK I.

High levels of ROCK I significantly correlated with shorter overall survival for patients compared with patients whose tumours exhibited low levels of ROCK I, while the difference in overall survival for patients with high levels of ROCK II was not significantly different from those with low levels of this transcript. These findings may point to ROCK I being a more potent kinase than ROCK II in breast tissue although, again, the balance of these two isoforms within the cell may be equally important in maintaining cytoskeletal integrity and so controlling tumour invasiveness.

Expression of ROCK proteins have been investigated in murine tissue with ROCK I and II showing different distributions within the body organs, although breast tissue was not examined (25). This study concluded that the ROCK proteins constitute a distinct kinase family of at least two isoforms. Ribozymes that target ROCK I have been shown to block invasive activity of tumour cells without affecting ROCK II expression thus indicating independent actions of ROCK I and ROCK II (26).

The ROCK knockdown cells, MDA-MB-231 ${ }^{\triangle R O C K I}$ and MDA-MB-231 $\triangle$ ROCKII did not show differences in their behaviour in relation to motility and invasion. Both demonstrated a significant decrease in invasiveness and migration compared with control cells, and this, together with a reduction in invasive response to $\mathrm{HGF}$ seen in the ROCK deficient cells, emphasises the important role played by these effector
A

Survival Function

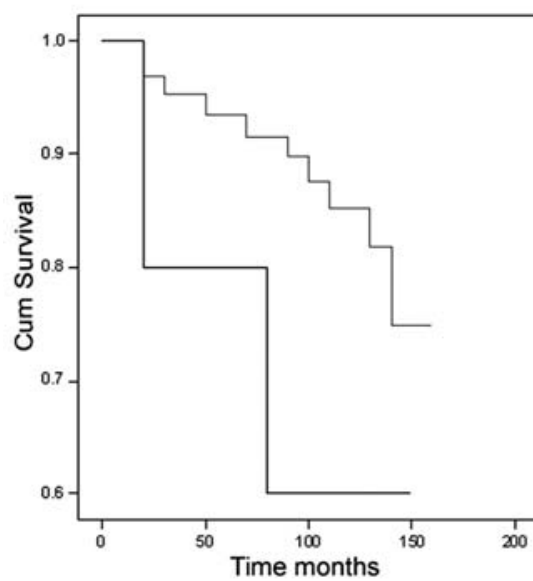

ROCK I

- ROCKIL

B Survival Function

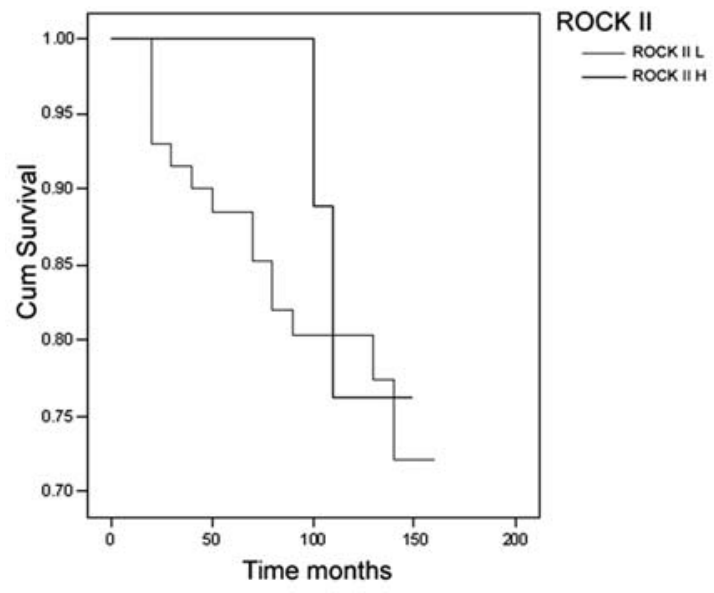

Figure 9. Kaplan-Meier survival curves. (A) Association of ROCK I expression with overall survival ( $\mathrm{p}=0.0304)$. (B) Association of ROCK II expression with overall survival $(\mathrm{p}=0.4550)$.

proteins in the motility and invasion of breast cancer cells. Negating the downstream effects of the ROCKs on cell contractility will have an important effect on cell migration and thus invasion. Addition of the ROCK inhibitor Y-27632 to the MDA-MB-231 GFP cells resulted in a decrease in invasiveness compared with untreated GFP cells, which correlates well with the results of work on a novel ROCK inhibitor Wf-536, which has been reported to inhibit in vitro invasion of B16 melanoma cells as well as in vivo suppression of tumour colony formation in the lung (27). Studies on Y-27632 and rat ascites hepatoma (MM1) cells, showed induced suppression of migration and morphological change of MM1 cells, thus suppressing tumour cell invasion (18). Therefore, ROCK inhibitors may be potential therapeutic agents in the treatment of tumour metastasis.

However, when looking at the MDA-MB-231 ${ }^{\triangle R O C K I}$ and MDA-MB-231 $\triangle$ RROCKII cells we see that knocking down either one of these molecules causes statistically decreased motility and invasion of these cells. This may indicate that it is the balance of these two isoforms that is important in maintaining cytoskeletal integrity within the cell and so preventing tumour 
growth and metastasis. It has been shown that only ROCK I and not ROCK II binds to Rho E, and that Rho E is a substrate for ROCK I and not ROCK II, thus indicating that these two highly homologous kinases have different targets (28).

It is noteworthy that the current study showed a similar in vivo tumour growth rate between ROCK manipulated cells and control cells. This indicates that ROCK activity may be primarily targeted at the regulation of cell motility and invasion, and has little impact on the growth of the breast tumours.

This study reports for the first time the increased expression of ROCK I in human breast cancer. Both ROCK I and ROCK II knockdown cells showed a significant decrease in motility and invasion which points to these kinases acting as key factors in the regulation of motility and invasion of breast cancer cells. This, together with the significant correlation between ROCK I, tumour aggression and survival in clinical breast cancer, indicates that it is a potential therapeutic target in human breast cancer, with the possible use of ROCK inhibitors acting as antimetastatic chemotherapeutic agents.

\section{Acknowledgements}

The authors wish to thank the Fong Family Foundation, Cancer Research Wales and the Emma Jane Demery Bequest Fund for supporting this work.

\section{References}

1. Hall A: Rho GTPases and the actin cytoskeleton. Science 11: 2295-2322, 1998.

2. Ridley AJ: Rho GTPases and cell migration. J Cell Sci 114: 2713-2722, 2001.

3. Takaishi K, Sasaki T, Kotani H, Nishioka H and Takai Y: Regulation of cell-cell adhesion by rac and rho small $\mathrm{G}$ proteins in MDCK cells. J Cell Biol 137: 1421-1431, 1997.

4. Jaffe $A B$ and Hall A: Rho GTPases in transformation and metastasis. Adv Cancer Res 84: 57-80, 2002.

5. Del Peso L, Hernandez-Alcoceba R, Embade N, Carnero A, Esteve P, Paje C and Lacal JC: Rho proteins induce metastatic properties in vivo. Oncogene 15: 3047-3057, 1997.

6. Kamai T, Tsujii T, Arai K, Takagi K, Asami H, Ito Y and Oshima H: Significant association of Rho/ROCK pathway with invasion and metastasis of bladder cancer. Clin Cancer Res 9: 2632-2641, 2003

7. Fritz G, Just I and Kaina B: Rho GTPase overexpressed in human tumours. Int J Cancer 81: 682-687, 1999.

8. Jiang WG, Watkins G, Lane J, Cunnick GH, Douglas-Jones A, Mokbel K and Mansel RE: Prognostic value of Rho GTPases and Rho guanine nucleotide dissociation inhibitors (GDI's) in human breast cancers. Clin Cancer Res 17: 6432-6440, 2003.

9. Bishop AL and Hall A: Rho GTPases and their effector proteins: Biochem J 348: 241-255, 2000.

10. Wang DS, Dou KF, Li KZ and Song ZS: Enhancement of migration and invasion of hepatoma cells via a Rho GTPase signaling pathway. World J Gastroenterol 10: 299-302, 2004.

11. Nishimura Y, Itoh K, Yoshioka K, Tokuda K and Himeno M: Overexpression of ROCK in human breast cancer cells: evidence that ROCK activity mediates intracellular membrane traffic of lysosomes. Pathol Oncol Res 9: 83-95, 2003.
12. Ishizaki T, Naito M, Fujisawa K, Maekawa M, Watanabe N, Saito Y and Narumiya S: p160ROCK, a Rho-associated coiledcoil forming protein kinase, works downstream of Rho and induces focal adhesions. FEBS Lett 404: 118-124, 1997.

13. Riento K and Ridley AJ: ROCKS: Multifunctional kinases in cell behaviour. Nat Rev Mol Cell Biol 4: 446-456, 2003.

14. Sahai E, Garcia-Medina R, Pouysségur J and Vial E: Smurf1 regulates tumor cell plasticity and motility through degradation of Rho A leading to localized inhibition of contractility. J Cell Biol 176: 35-42, 2007.

15. Torka R, Thuma F, Herzog V and Kirfel G: ROCK signaling mediates the adoption of different modes of migration and invasion in human mammary epithelial tumor cells. Exp Cell Res 312: 3857-3871, 2006.

16. Nakajima M, Hayashi K, Egi Y, Katayama K, Amano Y, Uehata M, Ohtsuki M, Fujii A, Oshita K, Kataoka H, Chiba K, Goto N and Kondo T: Effect of Wf-536, a novel ROCK inhibitor, against metastasis of B 16 melanoma. Cancer Chemother Pharmacol 52: 319-324, 2003.

17. Itoh $\mathrm{K}$, Yoshioka $\mathrm{K}$, Akedo $\mathrm{H}$, Uehata M, Ishizaki $\mathrm{T}$ and Narumiya S: An essential part for Rho-associated kinase in the transcellular invasion of tumour cells. Nat Med 5: 221-225, 1999.

18. Imamura F, Mukai M, Ayaki M and Akedo H: Y-27632, an inhibitor of Rho-associated protein kinase, suppresses tumour cell invasion via regulation of focal adhesion and focal adhesion kinase. Jpn J Cancer Res 91: 811-816, 2000.

19. Croft DR, Sahai E, Mavria G, Li S, Tsai J, Lee WM, Marshall CJ and Olson MF: Conditional ROCK activation in vivo induces tumor cell dissemination and angiogenesis. Cancer Res 64: 8994-9001, 2004.

20. Vishnubhotla R, Sun S, Huq J, Bulic M, Ramesh A, Guzman G, Cho $\mathrm{M}$ and Glover SC: ROCK-II mediates colon cancer invasion via regulation of MMP-2 and MMP-13 at the site of inva-dopodia as revealed by multiphoton imaging. Lab Invest 87: 1149-1158, 2007.

21. Kamai T, Arai K, Sumi S, Tsujii T, Honda M, Yamanishi T and Yoshida K: The rho-rho kinase pathway is involved in the progression of testicular germ cell tumour. BJU Int 89: 449-453, 2002.

22. Kamai T, Yamanishi T, Shirataki H, Takagi K, Asami H, Ito Y, Yoshida K: Overexpression of RhoA, Rac1 and Cdc42 GTPases is associated with progression in testicular cancer. Clin Cancer Res 10: 4799-4805, 2004.

23. Jiang WG, Davies G, Martin TA, Parr C, Watkins G, Mason MD, Mokbel K and Mansel RE: Targeting matrilysin and its impact on tumour growth in vivo: the potential implications in breast cancer therapy. Clin Cancer Res 11: 6012-6019, 2005.

24. Martin TA, Parr C, Davies G, Watkins G, Lane J, Matsumoto K, Nakamura T, Mansel RE and Jiang WG: Growth and angiogenesis of human breast cancer in a nude mouse tumour model is reduced by NK4, a HGF/SF antagonist. Carcinogenesis 24 : $1317-1323,2003$

25. Nakagawa O, Fujisawa K, Ishizaki T, Saito Y, Nakao K and Narumiya S: ROCK-I and ROCK-II, two isoforms of Rhoassociated coiled-coil forming protein serine/threonine kinase in mice. FEBS Lett 392: 189-193, 1996.

26. Suyama E, Kawasaki H, Kasaoka T and Taira K: Identification of genes responsible for cell migration by a library of randomized ribozymes. Cancer Res 63: 119-124, 2003.

27. Nakajima M, Hayashi K, Katayama K, Amano Y, Egi Y, Uehata M, Goto N and Kondo T: Wf-536 prevents tumor metastasis by inhibiting both tumor motility and angiogenic actions. Eur J Pharmacol 459: 113-120, 2003.

28. Riento K, Totty N, Villalonga P, Garg R, Guasch R and Ridley AJ: Rho E function is regulated by ROCK I-mediated phosphorylation. EMBO J 24: 1170-1180, 2005. 